\title{
El intervalo de tiempo entre la estimulación ovárica con FSH/LH y la colecta afecta la cantidad, calidad y capacidad de desarrollo de los ovocitos recuperados de novillas criollas ecuatorianas
}

\author{
The time interval between ovarian stimulation with FSH/LH and ovum-pick up \\ affects the quantity, quality and developmental competence of oocytes retrieved \\ from Ecuadorian creole heifers
}
Luis Ayala Guanga ${ }^{1}$, Jorge Samaniego Campoverde ${ }^{2}$, Daniel Argudo Garzón' ${ }^{1}$, Mariana Perea Brugal ${ }^{3}$, Fernando Perea Ganchou ${ }^{1,4}$, Ermes Rodas Carpio ${ }^{1,5}$, Pedro Nieto Escandón ${ }^{1}$

\section{Resumen}

\begin{abstract}
El objetivo del estudio fue evaluar los efectos del tratamiento con gonadotropina y el intervalo entre la estimulación ovárica con FSH/LH y la sesión de aspiración folicular, sobre la cantidad y calidad de los complejos cúmulus ovocitos (COCs) obtenidos por ovum pick up (OPU) en vaquillas criollas ecuatorianas. Cuatro novillas fueron asignadas aleatoriamente a cuatro grupos (FHS/LH 40h; FSH/LH 48h; control 40h; control 48h). En dos grupos se aplicó una dosis $(0.011 \mathrm{mg}$, IM) de GnRH el día 0, seguida de 500 UI, IM, de FSH/LH el día 2, y la sesión de OPU a los 40 (FSH/LH 40 h) o 48 horas (FSH/LH 48h) después de la aplicación de la gonadotropina. En los otros dos grupos, la FSH/LH se reemplazó por una solución fisiológica. No hubo diferencia estadística en la tasa de recuperación de los COCs entre FSH/LH 40h y FSH/LH 48h, pero se obtuvo aproximadamente el doble de $\mathrm{COCs}(\mathrm{p}<0.05)$ que en aquellos que no recibieron estimulación ovárica. Se consiguió 2.4 veces más COCs de calidad A en el tratamiento de FSH/LH 48h que en el de FSH/LH 40h. El 100\% de estas estructuras reaccionaron positivamente al Azul Brillante de Cresilo ( $\mathrm{BCB}+$ ), lo que significa que terminaron el crecimiento y fueron competentes para iniciar la maduración in vitro. Se concluye que el tratamiento con FSH/LH $48 \mathrm{~h}$ permitió obtener un mayor número de COCs competentes para la maduración in vitro.
\end{abstract}

Palabras clave: COCs, estimulación ovárica, aspiración folicular, Azul Brillante de Cresilo

\footnotetext{
${ }^{1}$ Carrera de Medicina Veterinaria Zootecnia, Facultad de Ciencias Agropecuarias, Universidad de Cuenca, Ecuador

${ }^{2}$ Laboratorio de Biotecnología, Facultad de Ciencias Agropecuarias, Universidad de Cuenca, Ecuador

${ }^{3}$ Facultad de Ciencias Veterinarias, Universidad del Zulia, Venezuela

${ }^{4}$ Departamento de Ciencias Agrarias, Universidad de Los Andes, Trujillo, Venezuela

${ }^{5}$ E-mail: ramiro.rodasc@ucuenca.edu.ec
}

Recibido: 3 de abril de 2019

Aceptado para publicación: 18 de enero de 2020

Publicado: 31 de marzo de 2020 
The aim of this study was to evaluate the effects of gonadotropin treatment and the interval between ovarian stimulation with $\mathrm{FSH} / \mathrm{LH}$ and the follicular aspiration session, on the quantity and quality of the cumulus oocyte complexes (COCs) obtained by ovum pick up (OPU) ) in Ecuadorian Creole heifers. Four heifers were randomly assigned to four groups (FHS/LH 40h; FSH/LH 48h; control 40h; control 48h). In two groups a dose (0.011 $\mathrm{mg}$, IM) of GnRH was applied on day 0 , followed by $500 \mathrm{IU}$, IM, of FSH/LH on day 2 , and the OPU session at 40 (FSH/LH $40 \mathrm{~h}$ ) or 48 hours (FSH/LH $48 \mathrm{~h}$ ) after the application of gonadotropin. In the other two groups, FSH/LH was replaced by a physiological solution. There was no statistical difference in the recovery rate of COCs between FSH/LH $40 \mathrm{~h}$ and FSH/LH 48h, but approximately twice as many COCs $(\mathrm{p}<0.05)$ were obtained than in those who did not receive ovarian stimulation. In addition, 2.4 times more COCs of quality A were achieved in the treatment of FSH/LH $48 \mathrm{~h}$ than in that of FSH/LH $40 \mathrm{~h}$. All $(100 \%)$ of these structures reacted positively to Brilliant Cresyl Blue (BCB+), which means that they finished the growth and were competent to initiate in vitro maturation. It is concluded that the treatment with $\mathrm{FSH} / \mathrm{LH} 48 \mathrm{~h}$ allowed to obtain a greater number of COCs competent for in vitro maturation.

Key words: COCs, ovarian stimulation, follicular aspiration, Brillant Cresyl Blue

\section{INTRODUCCIÓN}

El ganado criollo del Ecuador, así como de otras regiones de Latinoamérica se adaptó a diversas condiciones ambientales, lo cual les permitió adquirir características importantes como rusticidad, adaptabilidad, buena fertilidad y resistencia a enfermedades (Méndez et al., 2002; Aguirre et al., 2011). Sin embargo, debido a que su aptitud productiva no está bien definida, estas poblaciones han sido reemplazadas o vienen siendo absorbidas por razas bovinas más especializadas (Zárate $e t$ al., 2010); factores que ha provocado que este tipo de animales se encuentre dentro del $20 \%$ de las razas autóctonas en peligro de extinción (FAO, 2000).

Como estrategia para la conservación de este valioso material genético, se ha venido estudiando diversos aspectos reproductivos del ganado criollo, tales como la dinámica folicular (Ayala et al., 2017), la congelación de semen (Méndez et al., 2017) y la producción de ovocitos y embriones (Chasombat et al., 2013). La transferencia de embriones (TE) de vacas superovuladas (SOV) es una biotecnología que presenta diversos inconvenientes debido al reducido número de embriones transferibles y la variabilidad de los resultados (Restrepo et al., 2011). Así, la aspiración folicular transvaginal guiada por ultrasonido (OPU), técnica descrita inicialmente por Pieterse et al. (1988) es una alternativa para obtener ovocitos de vacas y novillas, que combinado con la producción de embriones in vitro (PIV) se viene convirtiendo en una eficiente herramienta.

La OPU permite el uso de vacas donantes de ovocitos en un intervalo de tiempo más corto (De Roover et al., 2008), además, los ovocitos recuperados provienen de vacas de alto valor genético que pueden conducir a un mejor rendimiento productivo de las ganaderías (Nava y Hernández, 2005); sin embargo, para obtener un mayor número de gametos con OPU, es necesario realizar una estimulación hormonal previa (Ding et al., 2008). Originalmente, se utilizaron protocolos de superovulación similares a los emplea- 
dos para obtener embriones in vivo (De Roover et al., 2005). Blondin et al. (1997) y Chaubal et al. (2006) observaron que el uso de gonadotropinas mejoró el rendimiento, en términos de calidad y cantidad de ovocitos recuperados; por otro lado, Ruiz et al. (2013) observaron que estos protocolos produjeron un desarrollo asincrónico de ovocitos y folículos que afectaba la calidad de los complejos cumulus ovocitos (COCs) obtenidos, mientras que Restrepo et al. (2011) reportan que el uso de FSH y LH combinados podría promover la maduración simultánea del folículo y el ovocito, obteniendo gametos de mejor calidad.

Para optimizar este método, los investigadores han valorado factores biológicos y técnicos, como la estimulación hormonal, el momento de la aspiración folicular después de la administración hormonal y la raza del donante, todo lo cual puede afectar la calidad de los ovocitos (Bols et al., 2004). Sin embargo, el intervalo más apropiado entre la administración de gonadotropina y la OPU no es bien conocido. Es así que, el propósito de este estudio fue evaluar los efectos del tratamiento con gonadotropina y el intervalo entre la estimulación ovárica con $\mathrm{FSH} / \mathrm{LH}$ y OPU sobre la cantidad, la calidad y la competencia de ovocitos recuperados de las novillas criollas, en un sistema de alimentación basado en pasturas.

\section{Materiales y Métodos}

\section{Lugar del Estudio y Animales}

El estudio se llevó a cabo en el Laboratorio de Biotecnología de Reproducción Animal en la granja experimental Irquis de la Universidad de Cuenca, provincia del Azuay, Ecuador, a una altitud de $2671 \mathrm{msnm}$. Como donantes de ovocitos se utilizaron cuatro novillas criollas, sin problemas de salud, sin alteraciones reproductivas, con edad de $19.4 \pm$ 0.8 meses, condición corporal entre 3 y 3.5 en la escala de 1 a 5 (Edmonson et al., 1989).
Los animales eran alimentados en pastizales de kikuyo (Pennisetum clandestinum), ray grass (Lolium perenne), trébol rojo (Trifolium pretense) y trébol blanco (Trifolium repens).

\section{Diseño Experimental y Tratamientos}

Las novillas recibieron $0.011 \mathrm{mg}$, vía IM, de GnRH (Conceptal ${ }^{\circledR}$, Intervet, Países Bajos) el día 0 para generar el reinicio de la onda folicular y se distribuyeron en cuatro tratamientos $48 \mathrm{~h}$ después, donde recibieron en $\mathrm{T} 1$ y T2 $=500$ UI, vía IM, de FSH/LH (Pluset ${ }^{2}$, Laboratorios Calier, España), T3 y T4 = (controles) un volumen equivalente de solución salina estéril. La recolección de ovocitos por OPU se realizó en T1 (FSH/LH 40h) y en T3 (Control 40h) a las 40h, y en T2 (FSH/LH 48h) y T4 (Control 48h) a las 48h después de la inyección de FSH/LH o de la solución salina. Las sesiones de OPU se llevaron a cabo a las 09:00, una vez a la semana, de acuerdo con el horario y la secuencia indicada en el Cuadro 1, siempre por el mismo técnico. Cada vaquilla fue utilizada en los cuatro tratamientos en forma alternada.

\section{Ecografía y Aspiración Folicular Transvaginal}

Una semana antes de comenzar el estudio, se realizó un examen de ultrasonido transrectal para monitorear la ciclicidad ovárica mediante la presencia de un cuerpo lúteo. Para la aspiración folicular, se inyectó a las novillas xilazina al $2 \%(0.05 \mathrm{mg} / \mathrm{kg}$, IM; Dormi-Xyl®2, Agrovet Market Animal Health, Perú), luego se vació el contenido rectal, se limpió la vulva y el área perineal y se aplicó lidocaína al 2\% (Roxicaína ${ }^{\circledR} 2 \%$, Ropsohn Therapeutics, Colombia) por vía epidural en una dosis de $0.3 \mathrm{mg} / \mathrm{kg}$.

Los ovarios se visualizaron y todos los folículos $\geq 5 \mathrm{y} \leq 8 \mathrm{~mm}$ se aspiraron utilizando un escáner de ultrasonido portátil (Prosound 2, Aloka, Japón), equipado con un transductor sectorial de 5.0 MHz ensamblado en un so- 
Cuadro 1. Secuencia de tratamientos de estimulación ovárica aplicados a las vaquillas criollas para colectar ovocitos mediante aspiración transvaginal guiada por ultrasonido

\begin{tabular}{lcccccc}
\hline \multirow{2}{*}{ Tratamientos } & \multicolumn{7}{c}{ Semana } \\
\cline { 2 - 6 } & 1 & 2 & 3 & 4 & 5 & 6 \\
\hline FSH/LH (40h) & Vaq 1 & Vaq 2 & Vaq 3 & Vaq 4 & Vaq 1 & - \\
FSH/LH (48h) & Vaq 2 & Vaq 1 & Vaq 4 & Vaq 3 & Vaq 2 & - \\
Control (40h) & Vaq 3 & Vaq 4 & Vaq 2 & Vaq 1 & Vaq 3 & Vaq 4 \\
Control (48h) & Vaq 4 & Vaq 3 & Vaq 1 & Vaq 2 & Vaq 4 & Vaq 3 \\
\hline
\end{tabular}

porte de sonda, utilizado por vía transvaginal. Este último contenía la guía de punción conformada por una aguja hipodérmica desechable $(1.2 \times 75 \mathrm{~mm})$ conectada en un extremo a un tubo cónico de $50 \mathrm{ml}$, a través de un sistema de teflón y el otro a una bomba de aspiración al vacío (WTA BV 003D, Brasil) con una presión regulada a $70 \mathrm{mmHg}$. Se usó solución salina tamponada con fosfato (PBS) suplementada con antibiótico y heparina como medio de recolección de COCs, que se mantuvo a $37{ }^{\circ} \mathrm{C}$ mientras se realizaba la OPU. Inmediatamente después de la sesión, el contenido del tubo se pasó por un filtro EmCon (Zona ${ }^{\mathrm{TM}}$, Agtech, USA), y se transfirió a una placa Petri cuadriculada de $95 \mathrm{~mm}$. Bajo un estereoscopio (SMZ 745, Nikon, Japón), los COCs fueron identificados y clasificados morfológicamente según los criterios indicados por De Loos et al. (1989).

La calidad de los ovocitos fue determinada de la siguiente manera: Calidad A; apariencia compacta, con múltiples capas $(>4)$ de células de cúmulus, citoplasma granular uniforme y transparente; Calidad B: 1 a 3 capas de células de cúmulus que cubrían la zona pelúcida, con citoplasma opaco que era total o parcialmente homogéneo y finamente granulado; Calidad C: parcial o totalmente desnudados, citoplasma con áreas oscuras irregulares; Calidad D: células del cumulus completamente expandidas y decoloradas.

\section{Competencia de los COCs}

Para evaluar la actividad de la glucosa6-fosfato deshidrogenasa se utilizó la tinción del azul brillante de cresilo BCB (B-5388, Sigma-Aldrich Chemie, Alemania). Los ovocitos, previamente clasificados como A, $\mathrm{B}, \mathrm{C}$ o D, se incubaron a $38.5^{\circ} \mathrm{C}$ durante 90 min con 26 ìm de BCB diluido en fluido de oviducto sintético (H-SOF) en una atmósfera con $90 \%$ de humedad, como lo describe Rodríguez et al. (2002). Luego, los ovocitos se lavaron tres veces con H-SOF y se evaluaron con un estereoscopio para determinar si habían terminado (citoplasma teñido: $\mathrm{BCB}+$ ) o no (citoplasma no teñido: $\mathrm{BCB}-$ ) la fase de crecimiento.

\section{Análisis Estadístico}

Se trabajó con cuatro tratamientos y seis repeticiones; sin embargo, la sexta repetición de FSH/LH 40h y FSH/LH 48h fue eliminada por problemas suscitados en el protocolo. El tratamiento, el factor animal y la repetición fueron considerados como variables independientes; mientras que el número de folículos detectados en los ovarios, el número de COCs recuperados, el número de COCs de clase A, B, C o D y el número de ovocitos de clase $\mathrm{A}, \mathrm{B}, \mathrm{C}$ o D que reaccionaron positivamente a la tinción con $\mathrm{BCB}$ fueron considerados como variables dependientes. 
Cuadro 2. Número de folículos detectados por ecografía, COCs recuperados mediante ovum pickup (OPU) y porcentaje de recuperación en respuesta a dos protocolos de estimulación ovárica en vaquillas criollas (media por sesión \pm error estándar)

\begin{tabular}{lccccc}
\hline \multirow{2}{*}{ Tratamientos } & \multicolumn{2}{c}{ Folículos visualizados } & \multicolumn{3}{c}{ COCs recuperados } \\
\cline { 2 - 6 } & $\mathrm{n}$ & Media \pm E.E. & $\mathrm{n}$ & Media \pm E.E. & $\%$ \\
\hline FSH/LH (40h) & 51 & $10.2 \pm 0.98^{\mathrm{a}}$ & 41 & $8.2 \pm 0.89^{\mathrm{a}}$ & $80.4^{\mathrm{a}}$ \\
FSH/LH (48h) & 61 & $12.2 \pm 0.98^{\mathrm{a}}$ & 49 & $9.8 \pm 0.89^{\mathrm{a}}$ & $80.3^{\mathrm{a}}$ \\
Control (40h) & 36 & $6.0 \pm 0,89^{\mathrm{b}}$ & 21 & $3.5 \pm 0.81^{\mathrm{b}}$ & $58.3^{\mathrm{b}}$ \\
Control (48h) & 36 & $6.0 \pm 0,89^{\mathrm{b}}$ & 24 & $4.0 \pm 0.81^{\mathrm{b}}$ & $66.7^{\mathrm{b}}$ \\
\hline
\end{tabular}

a,b Valores con letras diferentes en la misma columna indican diferencia significativa $(p<0.05)$

Cuadro 3. Número de COCs recuperados de acuerdo con el tratamiento de estimulación ovárica y la calidad ovocitaria (media \pm error estándar)

\begin{tabular}{ccccccc}
\hline \multirow{2}{*}{ Tratamientos } & \multicolumn{7}{c}{ Calidad de los ovocitos } \\
\cline { 2 - 7 } & $\mathrm{A}$ & $\mathrm{B}$ & $\mathrm{A}+\mathrm{B}$ & $\mathrm{C}$ & $\mathrm{D}$ & $\mathrm{C}+\mathrm{D}$ \\
\hline FSH/LH (40h) & $1.4 \pm 0.45^{\mathrm{b}}$ & $3.2 \pm 0.81^{\mathrm{a}}$ & $4.6 \pm 0.93^{\mathrm{a}}$ & $2.2 \pm 0.64$ & $1.4 \pm 0.44^{\mathrm{ab}}$ & $3.6 \pm 0.83$ \\
FSH/LH (48h) & $3.4 \pm 0.45^{\mathrm{a}}$ & $3.0 \pm 0.81^{\mathrm{a}}$ & $6.4 \pm 0.93^{\mathrm{a}}$ & $1.6 \pm 0.64$ & $1.8 \pm 0.44^{\mathrm{a}}$ & $3.4 \pm 0.83$ \\
Control (40h) & $0.4 \pm 0.45^{\mathrm{c}}$ & $0.8 \pm 0.81^{\mathrm{b}}$ & $1.2 \pm 0.85^{\mathrm{c}}$ & $1.8 \pm 0.64$ & $0.5 \pm 0.40^{\mathrm{b}}$ & $2.3 \pm 0.76$ \\
Control (48h) & $0.2 \pm 0.45^{\mathrm{c}}$ & $0.4 \pm 0.81^{\mathrm{b}}$ & $0.6 \pm 0.85^{\mathrm{c}}$ & $2.0 \pm 0.64$ & $0.5 \pm 0.40^{\mathrm{b}}$ & $2.5 \pm 0.76$ \\
\hline
\end{tabular}

$a, b$ Valores con letras diferentes en la misma columna indican diferencia significativa $(p<0.05)$

Calidad A: apariencia compacta, con múltiples capas $(>4)$ de células de cúmulus, citoplasma granular uniforme y transparente; Calidad B: 1 a 3 capas de células de cúmulus que cubrían la zona pelúcida, con citoplasma opaco que era total o parcialmente homogéneo y finamente granulado; Calidad C: parcial o totalmente desnudados, citoplasma con áreas oscuras irregulares; Calidad D: células del cumulus completamente expandidas y decoloradas

Los datos se examinaron mediante análisis de varianza utilizando el procedimiento del Modelo lineal general (GLM) de SAS para los efectos principales del tratamiento, el tiempo de recolección y la interacción. Las diferencias entre las medias se compararon mediante la prueba de Tukey-Kramer. Los valores de probabilidad de 0.05 o menos se consideraron significativos.

\section{Resultados}

\section{Folículos Detectados y COCs Recupe- rados}

El Cuadro 2 muestra la relación entre el número de folículos detectados por ultrasonido y el número de ovocitos recuperados por 
OPU. Como se esperaba, en los protocolos con $\mathrm{FSH} / \mathrm{LH}$ se obtuvo un mayor número de ovocitos en comparación con aquellos en los que se utilizó solución salina como control. Por otro lado, en el Cuadro 3 se observa que la comparación de ovocitos recuperados por tratamiento e intervalo mostró un efecto principal del tratamiento $(\mathrm{p}<0.05)$. Se obtuvieron 4.7 y 5.8 ovocitos más en FSH/LH $40 \mathrm{~h}$ y $\mathrm{FSH} / \mathrm{LH} 48 \mathrm{~h}$ que en los grupos de control respectivos. Del mismo modo, las tasas de recuperación de ovocitos para $\mathrm{FSH} / \mathrm{LH} 40 \mathrm{~h}$ y FSH/LH $48 \mathrm{~h}$ fueron 22.1 y 13.6 puntos porcentuales mayores que su control respectivo $(\mathrm{p}<0.05)$. El intervalo y la interacción de intervalo del tratamiento no difirieron.

Los resultados obtenidos muestran que, independientemente del tratamiento, la estimulación ovárica con $\mathrm{FSH} / \mathrm{LH}$ permitió recuperar 2.4 veces más COCs que los tratamientos que no recibieron estimulación hormonal $(\mathrm{p}<0.05)$.

\section{Calidad de los Ovocitos}

Se tuvo un efecto principal del tratamiento $(p<0.01)$ e interacción $(p<0.05$; Cuadro 3) para los COCs de calidad A. Se obtuvieron más COCs de mejor calidad cuando se realizó la OPU a las 48 h $(3.4 \pm 0.45)$ que a las $40 \mathrm{~h}(1.4 \pm 0.45)$ después de la inyección de FSH/LH; es decir, en la FSH/LH 48 h se obtuvieron 2.4 veces más ovocitos de clase A que en FSH/LH 40h y casi 7 veces más que las obtenidas por el control $48 \mathrm{~h}$.

En el caso de los COCs de calidad B, la estimulación con gonadotropina permitió obtener alrededor de cinco ovocitos más que en los grupos control $(\mathrm{p}<0.05)$; así mismo, no hubo efecto principal de intervalo o interacción (Cuadro 3). Los COCs de calidad C no difirieron significativamente con el tratamiento o el intervalo de recolección, mientras que los ovocitos de calidad D, solo los COCs recuperados del grupo FSH/LH $48 \mathrm{~h}$ fueron estadísticamente diferentes a los grupos testigo $(\mathrm{p}<0.05)$.
Solo los COCs agrupados como A + B variaron con el tratamiento $(p<0.05)$, siendo más numerosos cuando las novillas fueron tratadas con FSH/LH que cuando no recibieron la estimulación con gonadotropina. Con FSH/LH 40h y FSH/LH 48h, se recuperaron 4 o 10 COCs más $(\mathrm{p}<0.05)$ que en los controles $40 \mathrm{~h}$ o $48 \mathrm{~h}$, respectivamente (Cuadro $3)$. Por otro lado, no hubo diferencias significativas entre tratamientos con los COCs agrupados como $\mathrm{C}+\mathrm{D}$.

\section{Respuesta de Ovocitos a la Prueba de BCB}

Como se indicó anteriormente, el tratamiento FSH/LH $48 \mathrm{~h}(3.4 \pm 0.45)$ no solo proporcionó en promedio 2 COCs de tipo A+ $(\mathrm{p}<0.05)$ que el tratamiento $\mathrm{FSH} / \mathrm{LH} 40 \mathrm{~h}(1.4$ \pm 0.05 ) (Cuadro 3 ), sino que, además, el 100\% de estos ovocitos (tipo $\mathrm{A}$ ) reaccionaron positivamente a la prueba del BCB; es decir, se encontraban preparados funcionalmente para continuar con el proceso de maduración (Cuadro 4). Sin embargo, es importante resaltar que si bien todos los ovocitos clasificados como de tipo A en los cuatro tratamientos fueron $\mathrm{BCB}+$, la ejecución de la sesión de OPU a las $48 \mathrm{~h}$ permitió obtener mayor cantidad de ovocitos de excelente calidad morfológica y que han terminado su crecimiento.

Se encontró un efecto del tratamiento con relación a los COCs de calidad $\mathrm{B}$ $(\mathrm{p}<0.01)$, así como una interacción tratamiento-intervalo $(\mathrm{p}<0.05)$. Más ovocitos en el grupo $\mathrm{FSH} / \mathrm{LH} 48 \mathrm{~h}$ fueron $\mathrm{BCB}+$ en comparación con los otros tres grupos experimentales, los cuales fueron similares entre sí. Los COCs $\mathrm{BCB}+$ de calidad $\mathrm{C}$ y $\mathrm{D}$ fueron similares entre sí.

La prueba de $\mathrm{BCB}$ determinó que de los COCs (A + B), el 25.5\% (2.4/9.4) se obtuvo de FSH/LH 40h, el 61.7\% (5.8/9.4) de FSH/LH 48h, el 8.5\% (0.8/9.4) del Control 40h y 4.3\% (0.4/9.4) del control 48h (Cuadro 4). Además, el número de COCs $(\mathrm{A}+\mathrm{B})$ 
Cuadro 4. Número de ovocitos que reaccionaron positivamente al Azul Brillante de Cresilo (BCB) de acuerdo con el tratamiento y la calidad ovocitaria (media \pm error estándar)

\begin{tabular}{lcccccc}
\hline \multirow{2}{*}{ Tratamientos } & $\mathrm{7}$ & \multicolumn{7}{c}{ Calidad de los ovocitos } \\
\cline { 2 - 7 } & $\mathrm{A}$ & $\mathrm{B}$ & $\mathrm{A}+\mathrm{B}$ & $\mathrm{C}$ & $\mathrm{D}$ & $\mathrm{C}+\mathrm{D}$ \\
\hline $\mathrm{FSH} / \mathrm{LH}(40 \mathrm{~h})$ & $1.4 \pm 0.45^{\mathrm{b}}$ & $1.0 \pm 0.49^{\mathrm{b}}$ & $2.4 \pm 0.74^{\mathrm{b}}$ & $1.2 \pm 0.55$ & $0.8 \pm 0.40$ & $2.0 \pm 0.83$ \\
$\mathrm{FSH} / \mathrm{LH}(48 \mathrm{~h})$ & $3.4 \pm 0.45^{\mathrm{a}}$ & $2.4 \pm 0.49^{\mathrm{a}}$ & $5.8 \pm 0.74^{\mathrm{a}}$ & $1.4 \pm 0.55$ & $1.0 \pm 0.40$ & $2.4 \pm 0.83$ \\
$\mathrm{Control}(40 \mathrm{~h})$ & $0.4 \pm 0.45^{\mathrm{c}}$ & $0.4 \pm 0.49^{\mathrm{b}}$ & $0.8 \pm 0.68^{\mathrm{c}}$ & $1.2 \pm 0.55$ & $0.4 \pm 0.40$ & $1.8 \pm 0.75$ \\
Control (48h) & $0.2 \pm 0.45^{\mathrm{c}}$ & $0.2 \pm 0.49^{\mathrm{b}}$ & $0.4 \pm 0.68^{\mathrm{c}}$ & $0.8 \pm 0.55$ & $0.2 \pm 0.40$ & $1.0 \pm 0.75$ \\
\hline a,b Valores con letras diferentes en la misma columna indican diferencia significativa ( $\mathrm{p}<0.05)$ \\
Calidad A: apariencia compacta, con múltiples capas (>4) de células de cúmulus, citoplasma granular \\
uniforme y transparente; Calidad B: 1 a 3 capas de células de cúmulus que cubrían la zona pelúcida, con \\
citoplasma opaco que era total o parcialmente homogéneo y finamente granulado; Calidad C: parcial o \\
totalmente desnudados, citoplasma con áreas oscuras irregulares; Calidad D: células del cumulus \\
completamente expandidas y decoloradas
\end{tabular}

que fueron $\mathrm{BCB}+$ fue aproximadamente 2.4 (5.8/2.4) veces más numeroso en $\mathrm{FSH} / \mathrm{LH}$ $48 \mathrm{~h}$ que en $\mathrm{FSH} / \mathrm{LH} 40 \mathrm{~h}$, y proporcionó entre 7 o 14 veces más COCs $\mathrm{BCB}+$ que cualquier tratamiento control ( $40 \mathrm{~h}$ y $48 \mathrm{~h}$ ). Por otro lado, ni el tratamiento ni el intervalo tuvieron efecto sobre los COCs $(\mathrm{C}+\mathrm{D})$ (Cuadro 4).

\section{Discusión}

\section{Folículos Detectados y COCs Recupe- rados}

Las tasas de recuperación de COCs (Cuadro 1) en los protocolos que incluían $\mathrm{FSH} / \mathrm{LH}$ fueron similares a las obtenidas por Chasombat et al. (2013) en novillas criollas tailandesas y por De Roover et al. (2008) en vacas Belgian Blue. Por otra parte, Chaubal et al. (2006) utilizando una dosis de $200 \mathrm{mg}$ de FSH recuperó una proporción menor de COCs $(66.3 \%)$ que la obtenida en este estudio. De manera similar, la aplicación de 500 UI de FSH/LH permitió recuperar porcentajes de COCs que variaron entre $56.7 \%$ (Ruiz et al., 2013) y 63.3\% (García et al., 2012).
El alto porcentaje de recuperación de COCs en la presente investigación, así como en otras, denota la eficiencia alcanzada con la OPU para obtener ovocitos de animales genéticamente valiosos. La diferencia entre los resultados de este estudio y los indicados anteriormente podría ser explicada por el protocolo de estimulación usado, la raza, experiencia del operador y el sistema de manejo de los animales utilizados (Pieterse et al., 1988; De Roover et al., 2005; Torres-Júnior et al., 2008).

De Roover et al. (2005) señalaron que un animal estimulado hormonalmente antes de la OPU aumenta significativamente la producción de folículos aspirados y COCs recuperados con respecto a aquellos animales que no recibieron dicha estimulación, tal como lo demuestran los resultados de este estudio. Diversos autores (Blondin et al., 1997; Chaubal et al., 2006; De Roover et al., 2008; García et al., 2012; Chasombat et al., 2013; Ruiz et al., 2013) han atribuido a la FSH el incremento del número de folículos antrales y preovulatorios en los animales estimulados. Esta hormona, además de intervenir en el proceso de esteroideogénesis ovárica, creci- 
miento y maduración folicular, ayuda a los folículos subordinados a escapar de la atresia durante la etapa de desviación folicular (Forde et al., 2011).

En vacas sin estimulación ovárica, De Roover et al. (2008) encontraron valores similares de folículos aspirados y COCs recuperados $(7.1 \pm 3.9$ y $4.1 \pm 3.1$ respectivamente) a los del presente estudio. Sin embargo, en los tratamientos con estimulación con gonadotropina, el número de folículos aspirados (14.9 \pm 9.9$)$ y los COCs recuperados (11.8 \pm 8.2 ) fue mayor que los encontrados en la presente investigación; diferencias posiblemente atribuidas al uso de implantes de progesterona (3 $\mathrm{mg}$ de Norgestomet) antes de la administración de gonadotropina (De Roover et al., 2008). Es posible que el implante podría mejor la estimulación y sincronización del desarrollo folicular (Bo et al., 1995).

Chaubal et al. (2006), así mismo, detectaron más folículos $(16.0 \pm 5.0)$ y recuperaron más COCs $(10.6 \pm 4.5)$ luego del tratamiento hormonal que en este estudio. Esta discrepancia podría deberse que dichos autores eliminaron el folículo dominante antes de la OPU y al uso de la vía subcutánea para la administración de la FSH. Garcia y Salaheddine (1998) demostraron que la ablación del folículo dominante suprime el efecto de retroalimentación negativa del $17 \beta$ estradiol e inhibina producidos por los folículos en crecimiento sobre la FSH, y sincroniza la emergencia de una nueva onda folicular.

\section{Calidad de los Ovocitos}

El número de ovocitos de calidad A obtenidos (Cuadro 2) fueron similares a los descritos por Blondin et al. (2002), donde los COCs obtenidos $48 \mathrm{~h}$ de aplicar la FSH $\left(\right.$ Folltropin $\left.^{\circledR}\right)$ permitieron que los ovocitos completaran la maduración citoplasmática y alcanzaran una mayor competencia para su desarrollo posterior que los obtenidos a las $33 \mathrm{~h}$ de la aplicación de la FSH. Las evidencias indican que el tiempo entre la última apli- cación de FSH y la sesión de OPU tiene relación con el crecimiento de los folículos medianos a grandes, y disminución de los pequeños, permitiendo una mejor competencia de los ovocitos obtenidos (Blondin y Sirard, 1995). Un periodo de 44 o $66 \mathrm{~h}$ entre la última dosis de FSH y la recuperación de ovocitos mediante OPU en un protocolo superovulatorio fue mejor que $20 \mathrm{o} 96 \mathrm{~h}$ para la producción de embriones (Blondin et al., 2012). Asimismo, un mayor tiempo del ovocito en el ambiente folicular permite almacenar más ARNm y proteínas moleculares claves para potenciar su capacidad de desarrollo, lo cual no sucede con los ovocitos recuperados prematuramente y que probablemente carecen de la maquinaria transcripcional necesaria para su desarrollo (Adams et al., 1992; Vassena et al., 2003).

$\mathrm{Al}$ agrupar los $\mathrm{COCs}$ aptos $(\mathrm{A}+\mathrm{B})$ recuperados de vaquillas que recibieron estimulación hormonal (FSH/LH 40h; FSH/LH $48 \mathrm{~h}$ ), se pudo observar una cantidad proporcionalmente mayor de estos COCs frente a los obtenidos de aquellas que sirvieron de control (Cuadro 3). Esto concuerda con lo indicado por Goodhand et al. (2000) y Blondin et al. (2002), quienes describieron que la utilización de FSH y LH no solo permiten el reclutamiento y crecimiento de un mayor número de folículos (efecto de la FSH) sino que, además, ejerce un efecto favorable sobre la calidad de los COCs recuperados (efecto LH).

\section{Actividad Positiva al BCB}

Los resultados indicaron que el $100 \%$ de los ovocitos de calidad A en los cuatro tratamientos reaccionaron positivamente al $\mathrm{BCB}$ (Cuadro 4) y, por lo tanto, habían terminado su crecimiento. Con relación a este hallazgo, Pujol et al. (2004) y Adams et al. (1992) indicaron que los ovocitos de mayor calidad morfológica tienen una mejor actividad de crecimiento frente a los ovocitos de menor calidad. Sin embargo, hay que considerar que el tratamiento FSH-LH $48 \mathrm{~h}$ proporcionó mayor cantidad de ovocitos de es- 
tas características comparado con los otros tres tratamientos.

La diferencia en la respuesta al $\mathrm{BCB}$ entre los ovocitos de calidad B del tratamiento FSH/LH 48h (80\%) y los de FSH/LH 40h $(31.25 \%)$ puede atribuirse al tiempo transcurrido entre la administración de la FSH/LH y la realización del OPU, ya que ha sido demostrado que un intervalo de tiempo adecuado entre ambos eventos permitió obtener un grupo homogéneo de ovocitos con mayor competencia para avanzar a estadios posteriores de desarrollo (Blondin et al., 1997).

\section{Conclusiones}

- La estimulación ovárica con FSH/LH antes de la sesión OPU proporcionó 2.4 veces más COCs que aquellos sin administración de gonadotropina.

- La realización de la sesión de la OPU 48 horas después de la aplicación de una dosis de FSH/LH en comparación con la aplicación a las 40 horas permitió obtener más COCs de calidad A.

- Todos los COCs recuperados 48 horas después de que la inyección de FSH/LH habían terminado su crecimiento $(\mathrm{BCB}+)$ $\mathrm{y}$ estuvieron en condiciones de iniciar la fase de maduración in vitro (IVM).

\section{Agradecimientos}

Los autores agradecen a la Dirección de Investigación de la Universidad de Cuenca por el financiamiento para la ejecución de este proyecto. Además, reconocen el apoyo al Dr. Keith Inskeep, División de Ciencias Veterinarias y Animales de la Universidad de West Virginia, por la lectura crítica de este manuscrito y sus sugerencias.

\section{Literatura Citada}

1. Adams GP, Matteri RL, Kastelic JP, Ko JC, Ginther OJ. 1992. Association between surges of follicle-stimulating hormone and the emergence of follicular waves in heifers. J Reprod Fertil 94: 177188. doi: 10.1530/jrf.0.0940177

2. Aguirre L, Bermeo A, Maza D, Merino L. 2011. Estudio fenotípico y zoométrico del bovino criollo de la sierra media y alta de la región sur del Ecuador (RSE). Actas Iberoam Conserv Anim 1: 392-396.

3. Ayala L, Pesántez J, Rodas E, Méndez $S$, Soria M, Torres S, Váquez J, Pesántez E. 2017. Tamaño del folículo ovulatorio, cuerpo lúteo y progesterona sanguínea en vaquillas receptoras de embriones de tres razas en pastoreo en Ecuador. Rev Prod Anim 29: 65-72.

4. Blondin P, Sirard M. 1995. Oocyte and follicular morphology as determining characteristics for developmental competence in bovine oocytes. Mol Reprod Dev 41: 54-62. doi: 10.1002/mrd.1080410109

5. Blondin P, Guibault L, Sirard M. 1997. The time interval between FSH$\mathrm{P}$ administration and slaughter can influence the developmental competence of beer heifer oocytes. Theriogenology 48: 808-813. doi: 10.1016/s0093-691x(97)00303-8

6. Blondin P, Bousquet D, Twagiramungu H, Barnes F, Sirard MA. 2002. Manipulation of follicular development to produce developmentally competent bovine oocytes. Biol Reprod 66: 38-43. doi: 10.1095/biolreprod66.1.38

7. Blondin P, Vigneault C, Nivet al, Sirard M. 2012. Improving oocyte quality in cows and heifers-What have we learned so far? Anim Reprod Sci 9: 281-289.

8. Bo GA, Adams G, Caccia M, Martinez M, Pierson R, Mapletoft R. 1995. Ovarian follicular wave emergence after treatment with progestogen and estradiol in cattle. Anim Reprod Sci 39: 193-204. doi: 10.1016/0378-4320(95)01389-H

9. Bols PE, Leroy JL, Vanholder T, Soom $A .2004$. A comparison of a mechanical sector and a linear array transducer for ultrasound-guided transvaginal oocyte retrieval (OPU) in the cow. Therioge- 
nology 62: 906-914. doi: 10.1016/ j.theriogenology.2003.12.016

10. Chasombat J, Nagai T, Parnpai R, Vongpralub T. 2013. Ovarian follicular dynamics, ovarian follicular growth, oocyte yield, in vitro embryo production and repeated oocyte pick up in Thai native heifers undergoing superstimulation. Asian-Australasian J Anim 26: 488-500. doi: 10.5713/ajas.2012.12519

11. Chaubal SA, Molina JA, Ohlrichs CL, Ferre LB, Faber DC, Bols PE, Riesen JW, et al. 2006. Comparison of different transvaginal ovum pick-up protocols to optimize oocyte retrieval and embryo production over a 10 -week period in cows. Theriogenology 65: 1631-1648. doi: $10.1016 / j$.theriogenology.2005.07.020

12. De Loos F, van Vliet C, van Maurik $P$, Kruip TA. 1989. Morphology of immature bovine oocytes. Gamete Res 24: 197204. doi: 10.1002/mrd.1120240207

13. De Roover R, Bols PE, Genicot G, Hanzen C. 2005. Characterisation of low, medium and high responders following FSH stimulation prior to ultrasound-guided transvaginal oocyte retrieval in cows. Theriogenology 63: 1902-1913. doi: 10.1016/j.theriogenology.2004.08.011

14. De Roover R, Feugang J, Bols PE, Genicot G, Hanzen C. 2008. Effects of ovum pick-up frequency and FSH stimulation: a retrospective study on seven years of beef cattle in vitro embryo production. Reprod Domest Anim 43: 239-245. doi: 10.1111/j.14390531.2007.00873.x

15. Ding LJ, Tian H Bin, Wang JJ, Chen $J$, Sha HY, Chen JQ, Cheng GX. 2008. Different intervals of ovum pickup affect the competence of oocytes to support the preimplantation development of cloned bovine embryos. Mol Reprod Dev 75: 1710-1715.
16. Edmonson AJ, Lean IJ, Weaver LD, Farver T, Webster G. 1989. A body condition scoring chart for Holstein dairy cows. J Dairy Sci 72: 68-78. doi: 10.3168/ jds.S0022-0302(89)79081-0

17. [FAO] Organización de las Naciones Unidas para la Agricultura y la Alimentación. 2000. Una tercera parte de las razas de animales de granja corre peligro de extinción. [Internet]. Disponible en: http://www.fao.org/Noticias/2000/ 001201-s.htm

18. Forde N, Beltman ME, Lonergan P, Diskin M, Roche JF, Crowe MA. 2011. Oestrous cycles in Bos taurus cattle. Anim Reprod Sci 124: 163-169. doi: 10.1016/j.anireprosci.2010.08.025

19. Garcia A, Salaheddine M. 1998. Effects of repeated ultrasoundguided transvaginal follicular aspiration on bovine oocyte recovery and subsequent follicular development. Theriogenology 50: 575-585. doi: 10.1016/s0093691x(98)00162-9

20. García JR, Romero J, Astiz S, Poto A, Ruiz S. 2012. Eficacia de la ablación folicular y de la administración exógena de GnRH (Dalmarelin $\left.{ }^{\circledR}\right)$ sobre la producción y calidad ovocitaria en vacas de raza Murciano-Levantina. En: XVII Congreso Internacional ANEMBE de Medicina bovina. España.

21. Goodhand $K L$, Staines ME, Hutchinson JS, Broadbent PJ. 2000. In vivo oocyte recovery and in vitro embryo production from bovine oocyte donors treated with progestagen, oestradiol and FSH. Anim Reprod Sci 63: 145-158. doi: 10.1016/s0378-4320(00)00186-x

22. Méndez M, Serrano J, Ávila R, Rosas M, Méndez N. 2002. Caracterización morfométrica del bovino criollo mixteco. Arch Zootec 51: 217-221.

23. MéndezS, Galarza D, Argudo D, Bueno $H$, Iñiguez $C$, Soria $M$, Torres $C$, et al. 2017. Effect of two collection methods and two extenders on the 
freezability of semen of native bulls in Ecuador's Andean highlands. Anim Reprod Sci 14: 303.

24. Nava H, Hernández H. 2005. Aspiración folicular transvaginal. En: González C, Soto E (eds). Manual Ganadería Doble Propósito. Maracaibo: Astro Data. p 611-614.

25. Pieterse $M$, Kappen K, Kruip A, Taverne M. 1988. Aspiration of bovine oocytes during transvaginal ultrasound scanning of the ovaries. Theriogenology 30: 751-762. doi: 10.1016/0093-691x(88)90310-x

26. Pujol M, López M, Paramio M. 2004. Developmental competence of heifer oocytes selected using the brilliant cresyl blue (BCB) test. Theriogenology 61: 735744. doi: 10.1016/s0093-691x(03)00250-4

27. Restrepo G, Gópmez J, Vasquez N. 2011. Evaluación de la superestimulación ovárica y la calidad morfológica de occitos bovinos obtenidos por aspiración folicular. Rev Politécnica 7: 16-21.

28. Rodríguez-González E, López-Béjar M, Velilla E, Paramio MT. 2002. Selection of prepubertal goat oocytes using the brilliant cresyl blue test.
Theriogenology 57: 1397-1409. doi: 10.1016/s0093-691x(02)00645-3

29. Ruiz S, Romero J, Astiz S, Peinado B, Almela L, Poto A. 2013. Application of reproductive biotechnology for the recovery of endangered breeds: birth of the first calf of Murciana-Levantina bovine breed derived by OPU, in vitro production and embryo vitrification. Reprod Domest Anim 48: e81-4. doi: 10.1111/rda.12179

30. Torres-Júnior J, Pires $M$, de Sá W, Ferreira A, Viana J, Camargo L, Ra$\operatorname{mos} A$, et al. 2008. Effect of maternal heat-stress on follicular growth and oocyte competence in Bos indicus cattle. Theriogenology 69: 155-166. doi: 10.1016/j.theriogenology.2007.06.023

31. Vassena R, Mapletoft R, Allodi S, Singh J, Adams G. 2003. Morphology and developmental competence of bovine oocytes relative to follicular status. Theriogenology 60: 923-932. doi: 10.1016/s0093-691x(03)00101-8

32. Zárate-Martínez J, Ramírez J, Rodríguez F. 2010. Comportamiento reproductivo de vacas criollas con amamantamiento restringido y sincronización del estro. Agron Mesoam 21: 121-130. 